\title{
Caracterización de la Infección por el Virus de la Inmunodeficiencia Humana en el Hospital Hermanos Ameinjeiras, 2002 - 2007
}

\section{Characterization of human immunodeficiency Virus infection in the Hospital}

Ameinjeiras Brothers, 2002-2007

Lourdes María Velázquez Lores ${ }^{1}$, Grey Marilú Andrade Tacuri ${ }^{2}$, Gabriela Gricel Triana Santana $^{3} \&$ Joselyn Aideé Ayala Briones ${ }^{4}$

Recibido: 10-02-2019 / Revisado: 15-02-209 /Aceptado: 04-03-2019/ Publicado: 27-04-2019

\begin{abstract}
.
DOI: https://doi.org/10.33262/cienciadigital.v3i2.1.437

Human immunodeficiency virus infection is a global epidemic that affects more than 40 million people worldwide, with a high rate of morbidity and mortality due to opportunistic diseases associated with advanced stages of the disease. In Cuba the incidence of this infection is $9.9 \times 100000$ inhabitants and the prevalence of $0.09 \%$, being the lowest records in the Caribbean area.
\end{abstract}

A retrospective, observational and descriptive study was conducted to know the clinical and epidemiological characteristics of the group of patients diagnosed with the human immunodeficiency virus between 2002 and 2007 in the Hermanos Amejjeiras hospital.

In the center, 80 cases were diagnosed that were confirmed in the reference laboratory (LISIDA), 58 on an outpatient basis and 22 admitted. In 2007, 25\% of the patients in

\footnotetext{
${ }^{1}$ Licenciada en Enfermeria, Magister en enfermedades infecciosas, Hospital Sagrado Corazón de Jesús. Email: lourdes.velazquez@distrito12d03.saludzona5.gob.ec. ulyargelia @ gmail .com

${ }^{2}$ Licenciada en enfermería, Magister enfermeria quirúrgica. Universidad Técnica de Babahoyo Email: grey.andrade@distrito12d03.saludzona5.gob.es lic-grey-andrade@hotmail.com

${ }^{3}$ Obstetra, diplomado en ecografia gineco-obsterica. Hospital Sagrado Corazón de Jesús. Email: gabriela.triana@distrito12d03.saludzona5.gob.es

${ }^{4}$ Licenciada en enfermería. Hospital Sagrado Corazón de Jesús. Email: joselynayalabri@gmail.com 
the sample were diagnosed, the period with the highest number of cases. The most important epidemiological elements were: the male sex and the origin of the City of Havana. The most frequent clinical symptoms were: lymphadenopathy (58.3\%), weight loss $(58.3 \%)$, asthenia (50\%) and diarrhea (41.3\%). Nine patients with acute retrovirosis were diagnosed, 9 with AIDS stage and 4 with asymptomatic carrier.

The diseases related to AIDS that were diagnosed in this series were: Pulmonary tuberculosis (2 cases), HIV wasting syndrome (2 cases), Burkitt high-grade nonHodgkin lymphoma (1 case), disseminated candidiasis (1 case), Pneumocystis carinii pneumonia ( 2 cases) and cerebral toxoplasmosis ( 1 cases).

Keywords: HIV I, HIV II, AIDS, Seropositive, TCD4 lymphocytes, Elisa

\section{Resumen.}

La infección por el virus de la inmunodeficiencia humana es una epidemia mundial que afecta a más de 40 millones de personas en todo el planeta, con un alto índice de morbilidad y mortalidad por enfermedades oportunistas asociadas a estadios avanzados de la enfermedad. En Cuba la incidencia de esta infección es de 9.9 x 100000 habitantes y la prevalencia de $0.09 \%$, siendo los registros más bajos del área del Caribe.

Se realizó un estudio retrospectivo, observacional y descriptivo para conocer las característica clínicas y epidemiológicas del grupo de pacientes diagnosticados con el virus de la inmunodeficiencia humana entre los años 2002 al 2007 en el hospital Hermanos Amejjeiras.

Se diagnosticaron en el centro 80 casos que fueron confirmados en el laboratorio de referencia (LISIDA), 58 de forma ambulatoria y 22 ingresados. En el año 2007 se diagnosticaron el $25 \%$ de los pacientes de la muestra, siendo el periodo de mayor número de casos. Los elementos epidemiológicos de mayor importancia fueron: el sexo masculino y la procedencia de la Ciudad de la Habana. Los sín- tomas clínicos más frecuentes fueron: linfadenopatías (58.3\%), pérdida de peso (58.3\%), astenia (50\%) y diarreas $(41.3 \%)$. Se diagnosticaron 9 pacientes con retrovirosis aguda, 9 en estadio SIDA y 4 como hallazgo en periodo de portador asintomático.

Las enfermedades relacionadas con SIDA que se diagnosticaron en esta serie fueron: La tuberculosis pulmonar (2 casos), el síndrome de desgaste por VIH (2 casos), Linfoma no Hodgkin de alto grado tipo Burkitt (1 caso), candidiasis dise- minada (1 caso), Neumonía por Pneumocistys carinii (2 casos) y toxoplasmosis cerebral (1 casos). 
Palabras claves: VIH I, VHI II, SIDA, Seropositivos, Linfocitos TCD4, Elisa

\section{Introducción.}

El Síndrome de Inmunodeficiencia Adquirida (SIDA) se diagnosticó por primera vez al inicio de la década de los 80 del siglo xx y su agente causal, un virus de la familia de los retrovirus, y fue aislado en 1982, tomándose uno de los azotes más temibles de la humanidad en los últimos veinte años. Hasta la fecha, 40 millones de personas en el mundo han adquirido la infección y 16 millones han fallecido después de dos décadas de estudio y manejo de pacientes con infección por el VIH se ha avanzado notablemente en el conocimiento del agente causal, los modelos de la enfermedad, estrategias de prevención y tratamiento. La vigilancia epidemiológica debe concentrarse en los grupos con conductas de riesgo y la ne pesquisa de casos nuevos debe ser continua con el objetivo fundamental de detectar pacientes en estadios iniciales de la infección lo que permite llevar a cabo acciones de prevención y brindar una atención médica adecuada. La epidemia cubana se ha caracterizado por.

- Detección inicial de personas que adquirieron la infección en el extranjero y sus parejas sexuales en cuba, dando inicio a la transmisión autóctono.

- Identificación de un foco de transmisión entre hombres que tienen sexo con hombres (hsh) en la región central del país, que aportó una importante cantidad de casos del sexo masculino y que inicia la transmisión sexual entre individuos con conducta sexual de riesgo.

- Progresiva detección de individuos infectados, que, según estudios realizados, pertenecen a grupos vulnerables con conducta sexual y social de riesgo, con cambios frecuente de parejas y muy baja percepción del riesgo.

- Es una epidemia de baja transmisión, autóctona y con una mayor detección en grupos nucleares las mayores tasas de detección acumulada se observan en provincias centrooccidentales

El Hospital brinda atención a una gran parte de la población de los municipios centro habana y habana vieja, además de asumir el estudio de un número elevado de pacientes de otros municipios de la capital, donde las tasas de infección son superiores a la nacional" por lo que decidimos hacer un estudio sobre el estado de la infección VIH/SIDA en el centro entre los años 2002- 2007 a fin de evaluar la importancia y frecuencia del diagnóstico en un nivel terciario de atención y trazar nuevas perspectivas en el trabajo de pesquisaje y manejo de esta infección. 


\section{Marco Teórico}

El síndrome de inmunodeficiencia humana (VIH), está definido por una ser de enfermedades oportunistas asociadas a la inmunodepresión que están relacionadas con el efecto que produce el virus de inmunodeficiencia humana (VIH), en el sistema inmunológico.

El (VIH), infecta al organismo mediante tres vías; las relaciones sexuales desprotegidas, la exposición directa de piel no intacta y mucosas a sangres contaminadas u otros derivados, dentro de los que tenemos el intercambio de jeringuillas y agujas, y la exposición ocupacional, y la tercera vía es la madre infectando al feto durante el embarazo, en el momento del parto, u a través de la lactancia materna, conocida como trasmisión materno infantil o trasmisión vertical.

El virus de inmunodeficiencia humana (VIH), es un retrovirus humano que pertenece al género de los lentivirus, con la característica de infectar y replicarse en una amplia de células humanas del sistema inmunológico, donde se encuentran los linfocitos TCD4, el sistema monocitomacrofogo, y microglias en el sistema nervioso central, siendo la infección del linfocito TCD4 (auxiliador y reparador), la que produce los efectos más catastróficos en las respuestas inmunológicas.

El linfocito TCD4, juega un papel central en la activación y mantenimiento de las respueta inmunológica, regula la respuesta de otras células que incluyen el complejo manocitomacrófago, células T citotoxicas (TCD8), las células B productoras de anticuerpos, que permite deducir el deterioro del funcionamiento o la disminución de cantidad del número del TCD4, trae consecuencias fatales para el sistema inmunitario del organismo humano, que conduce al individuo a las severa inmudeficiencia, que lo lleva a padecer las diferentes enfermedades oportunistas definitorias del SIDA,

La era del SIDA empezó el 5 de junio del año 1981, cuando el Center For Disease control and prevention (centro para la prevención y control de las enfermedades), de Estados Unidos convoco una conferencia de prensa donde describió 5 casos de neumonía por Pneumocytis Cariniis en los Angeles. Al mes siguiente se constataron varios de sarcomas de Kaposis, un tipo de cáncer de tipo de piel; las primeras constataciones de estos casos fueron realizadas por el doctor. Michael Gottlieb de Sa Francisco.

Pese a que los médicos conocían tanto la neumonía por Pneumocytis Cariniis, como el sarcoma de Kaposis, la aparición conjunta de ambos en varios pacientes les llamo la atención. La mayoría de los pacientes eran homosexuales sexualmente activos, muchos de los cuales sufrían de otras enfermedades crónicas que más tarde se identificaron como infecciones oportunistas. Las pruebas sanguíneas que se realizaron a estos pacientes mostraron que 
carecían del número adecuado de un tipo de células sanguíneas llamadas TCD4, gran parte de los pacientes murieron en pocos meses.

En un principio se identificó a la población homosexual, como víctima de la deficiencia inmune, la prensa comenzó a llamarla la peste rosa, aunque pronto se hizo notar que también la padecía los emigrantes Haitianos en los Estados Unidos, los usuarios de drogas intravenosas, los receptores de trasfusiones sanguíneas, y las mujeres heterosexuales; la nueva enfermedad fue bautizada oficialmente en el año 1982 con el nombre de Síndrome de inmunodeficiencia adquirida (SIDA).

\section{Metodologia.}

El algoritmo diagnóstico de la infección VIH/SIDA en nuestro país establecido por el Programa Nacional de Prevención y Control de la infección por el VIH y el SIDA se establece de la siguiente forma:

PROGRAMA DE PESQUISAJE > ELISA + LABORATORIO NACIONAL POSITIVO DE REFERENCIA e Donantes de sangre (2 MUESTRAS) (LISIDA) e Infecciones de transmisión sexual + Captados e Gestantes e Contactos de seropositivos ELISA e Sospecha clínica | o WESTERN BLOT

\section{REGISTRO NACIONAL DE SEROPOSITIVOS *POSITIVO}

A la primera muestra recogida en la Red Nacional de Laboratorios se le realiza UMELISA HIV 1-2 RECOMBINANTE, por el equipo SUMA. Este es un ensayo inmunoenzimático indirecto de segunda generación, para la detección de anti- cuerpos al VIH 1 y VIH 2, que emplea, como antígenos de captura a gp120, gp41 y p24, proteínas representativas de la envoltura y núcleo del virus VIH 1 así como el antígeno gp36, proteína de la envoltura del VIH 2, obtenidas por métodos re- combinantes. ***? Las muestras que resultan ELISA reactivas por segunda vez son enviadas al Laboratorio Nacional de Referencia donde la muestra es sometida a una prueba adicional de ELISA de distinto principio y antígeno. Si se repite la reactividad se efectúa el ensayo de Western Blot (WB) para la detección de anticuerpos para el VIH 1 y si éste es positivo, se solicita una segunda muestra para verificar el diagnóstico. Si el WB resulta negativo o indeterminado se realiza WB para VIH 2 y si es positiva se solicita una segunda muestra para confirmación. De ser negativo este ensayo para VIH 1 y 2 se concluye como negativo.

La muestra a estudiar fue la siguiente: Se tomaron todos los casos positivos de ELISA-VIH del Laboratorio de Micro- biología de nuestro hospital entre los años 2002 al 2007 basado en los registros que lleva este Laboratorio 
Se tomaron todos los casos confirmados en el Laboratorio de Referencia Na-cional procedentes del Hospital Hermanos Ameijeiras entre los años 2002 y 2007

A los casos que han sido diagnosticados durante un ingreso hospitalario, se le confeccionó un modelo de recolección de datos (Anexo 1) con vistas al estudio del cuadro clínicoepidemiológico en el momento del diagnóstico Con los datos obtenidos se procedió al análisis y relación de acuerdo a la importancia de los mismos a fin de reflejar una información detallada del estado de la infección VIH/SIDA manejada en el hospital Ameijeiras entre los años 2002-2007. El análisis estadístico se realizó por cálculos de la t de Students; además de utilizar el análisis descriptivo para la mayoría de las variables.

\section{Resultados.}

Durante los años analizados, entre 2002 y el 2007 el Hospital Hermanos Ameijei- ras hizo diagnóstico de infección por el virus de la inmunodeficiencia humana (VIH) en 80 pacientes. La distribución de casos por año de diagnóstico se muestra en el Gráfico 1, siendo el año 2007 el de mayor número, con 20 pacientes confirmados, para un 25\% del total de los casos, aunque desde el año 2003 el número total de pacientes diagnosticados superó en más de 100 casos el reporte de los años anteriores. De acuerdo a la estadística del país a partir del año 2003, se observó un incremento de la infección por el virus de la inmunodeficiencia, siendo más notable en el año 2007. El reporte anual de la Dirección Nacional de Epidemiología del MINSAP ha informado desde 1996 un lento crecimiento de los casos seropositivos al VIH, alcanzando la cifra de 942 nuevos diagnósticos durante el 2006 y 817 durante el año 2006 en el país, por lo que nuestros resultados coinciden con el registro nacional.

Tabla $\mathbf{N}^{\circ} 1$ Distribución de casos de infección por vih, por años.

\begin{tabular}{lll}
\hline \multicolumn{1}{c}{$\mathbf{N}^{\circ}$ Caso } & \multicolumn{1}{c}{ Año } & \multicolumn{1}{c}{$\%$} \\
\hline 7 & 2002 & 8.75 \\
7 & 2003 & 8,75 \\
12 & 2004 & 15 \\
15 & 2005 & 18.75 \\
19 & 2006 & 23.75 \\
20 & 2007 & 25 \\
80 & Total & $100 \%$ \\
\hline
\end{tabular}

Fuente: Elaboración propia

Figura 1. Distribución de casos de infección por vih, por años. 


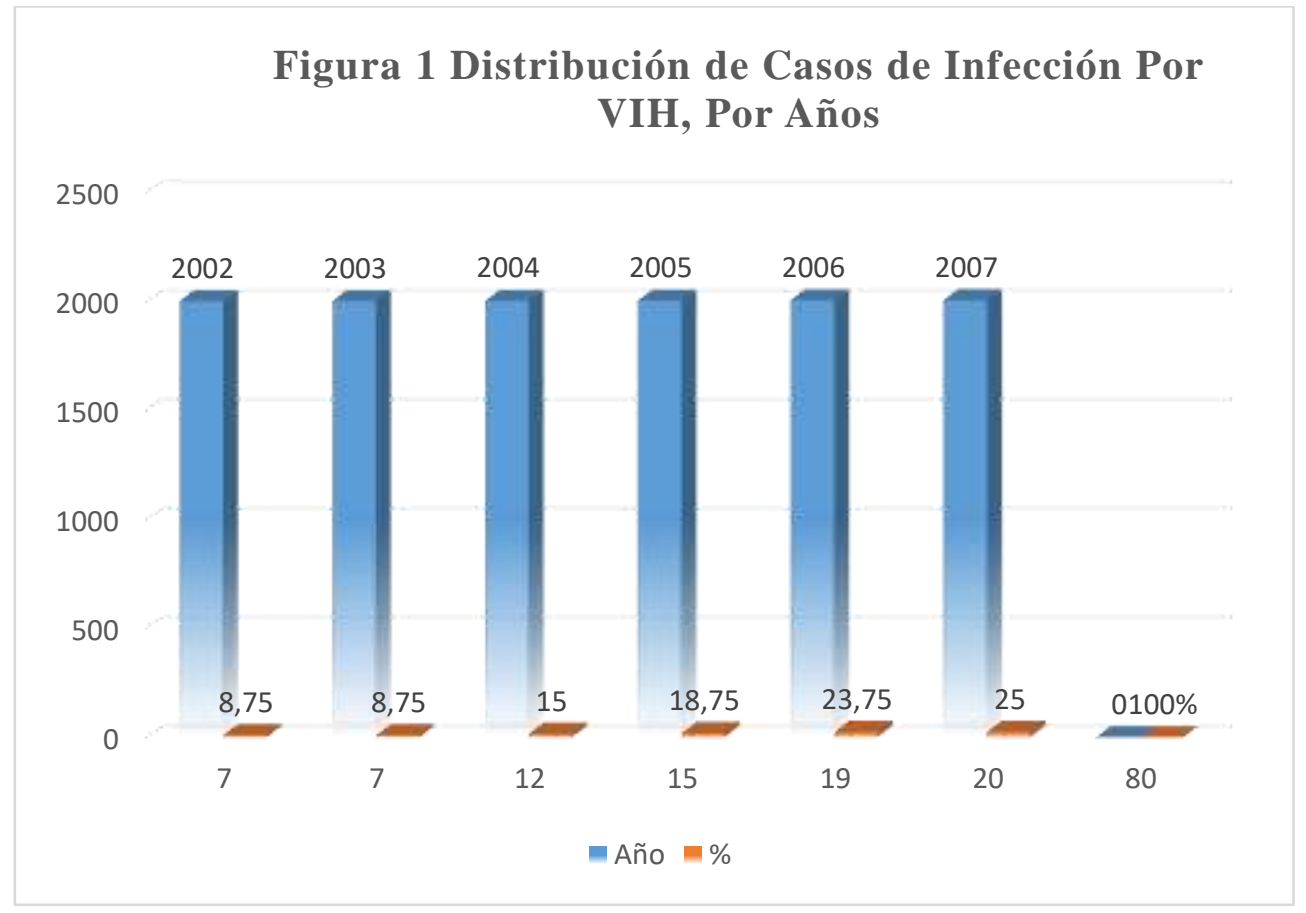

Fuente: Elaboración propia

\section{Análisis}

En la figura1 distribución de casos de infección por VIH, se observa un incremento de la infección por el virus de la inmunodeficiencia, siendo más notable en el año 2007, asimismo se evidencia que el comportamiento de este incremento en números de casos como promedio de un año a otro año es de 2 casos, que representan el 2, 5\%, el mencionado aumento de casos por infección del VIH, fue a partir del año 2003.

Del total de pacientes diagnosticados, 22 (27.5\%) fueron en el curso de un ingreso, 14 en el servicio de Medicina Interna, 1 en Quemados, 2 en Hematología, 2 en Dermatología, 1 en ORL y 2 en Gastroenterología. Los 58 restantes procedían de las consultas externas del hospital, lo cual está en relación con que se manejan mayor número de pacientes por vía ambulatoria y es donde se realiza mejor pesquisaje de las enfermedades de transmisión sexual (ETS).

En el paciente hospitalizado por lo general el estudio se orienta a la patología por la que ingresa el paciente y la indicación de serología VIH se hace de acuerdo al pensamiento clínico del médico.

El Programa de Prevención y control de la infección por VIH y el SIDA de Cuba reportó en diciembre del 2007 que, del total de seropositivos del país, el $80.6 \%$ son masculinos y $85.7 \%$ de éstos son Hombres que tienen sexo con Hombres (HSH). 
Muestra serie registró un franco predominio del sexo masculino (Gráfico 2), con un total de 73 hombres, para un $91.25 \%$, y un total de sexo femenino de 7 mujeres que representa el $8.75 \%$, lo cual es lo esperado de acuerdo a las estadísticas nacionales. El dato de la preferencia sexual solo lo obtuvimos en los casos ingresados, ya que esto muchas veces es de difícil obtención y requiere una encuesta dirigida y realizada por personal adiestrado, lo cual no fue posible en los ambulatorios.

Tabla N² Distribución de Casos de VIH Por Sexo.

\begin{tabular}{lll}
\hline Sexo & Total & \% \\
\hline Masculino & 73 & 91.25 \\
Femenino & 7 & 8.75 \\
\hline
\end{tabular}

Fuente: Elaboración propia

Gráfico $\mathbf{N}^{\circ} 2$ Distribución de Casos de VIH por Sexo.

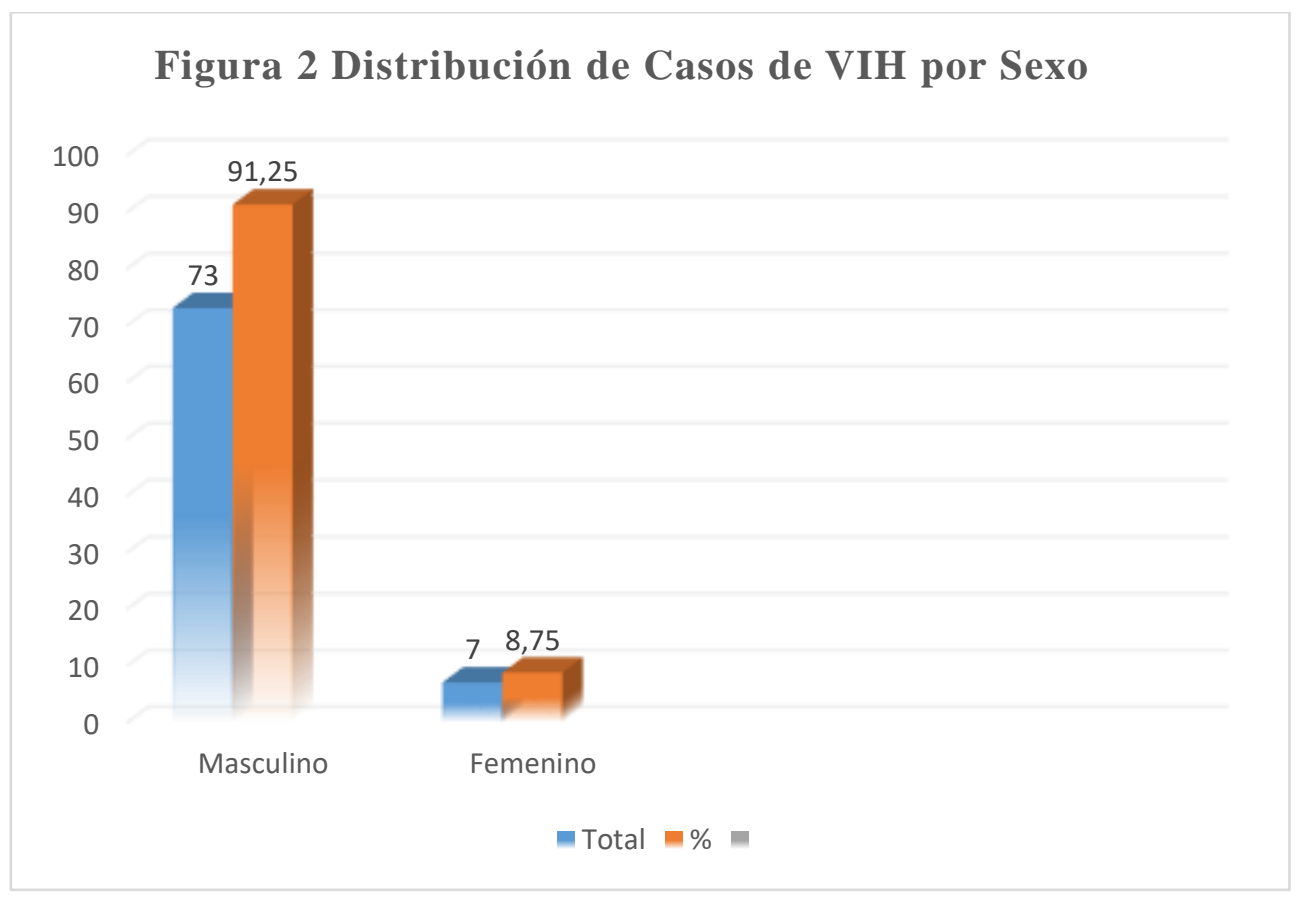

Fuente: Elaboración propia

\section{Análisis}

En la figura 2, registra un franco predominio del sexo masculino con un total de 73 hombres, que corresponde a un $91.25 \%$, y un total de sexo femenino de 7 mujeres que representa el $8.75 \%$, lo cual es lo esperado de acuerdo a las estadísticas nacionales. 
La cifra mayor de casos procedía de la Ciudad de La Habana (72, para un 90\%), las otras provincias reunieron un total de 8 seropositivos, La Habana con 4, Pinar del Río 1, Ciego de Ávila 1 y Camagúey 1 (Gráfico 3).

Tabla $\mathbf{N}^{\circ} 3$ Casos de Infección por VIH, Distribuido por Provincias.

\begin{tabular}{clcc}
\hline $\begin{array}{c}\text { Casos de infección } \\
\text { por VIH. }\end{array}$ & $\begin{array}{l}\text { Distribuido } \\
\text { Provincias }\end{array}$ & por & $\%$ \\
\hline 72 & Ciudad de & la & $90 \%$ \\
4 & Habana & $5 \%$ \\
1 & La Habana & $1.25 \%$ \\
1 & Pinard del Rio & $1.25 \%$ \\
1 & Ciego de Aguila & $1.25 \%$ \\
1 & Camaguey & $1.25 \%$ \\
\hline
\end{tabular}

Fuente: Elaboración propia

Figura 3. Casos de infección por vih, distribución por provincias

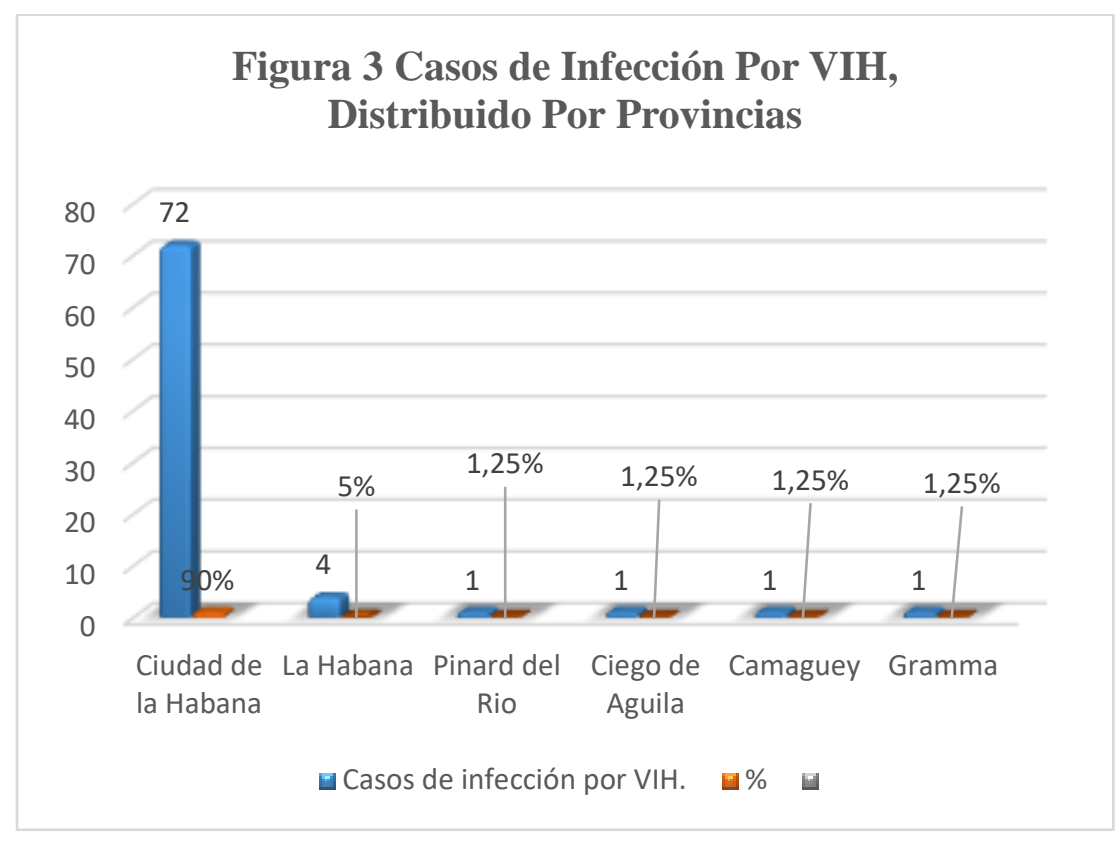

Fuente: Elaboración propia

\section{Análisis 3}

En la figura 3 se evidencia que la cifra mayor de casos procede de la Ciudad de La Habana, con número de 72 casos mismos que representan el $90 \%$. 
La tasa de infección acumulada para nuestro país es de 17.74 por 100000 habitantes, existen 26 municipios con tasas superiores a la nacional, siendo notables las tasas de Ciudad de La Habana (67\%), Santa Clara (67.2\%), Santiago de Cuba (61.8\%), Ciego de Ávila (54.5\%) y Pinar del Río (52.7\%). Y

El estudio de la historia clínica de los pacientes ingresados, nos permitió la obtención de los datos necesarios para el análisis de las manifestaciones clínicas que presentaron en el momento del diagnóstico, lo cual no fue posible en los casos ambulatorios, de los que solo tenemos datos generales, debido a que su confirmación y seguimiento se hizo por el Centro Provincial de Higiene y Epidemiología.

En los 22 pacientes estudiados la edad promedio fue de 36 años y el tiempo medio de evolución desde el comienzo de los síntomas hasta la confirmación de la infección fue de 6 meses con un rango entre 1 y 18 meses. De ellos, 9 se diagnosticaron durante el periodo agudo de la enfermedad, 9 en estadio SIDA ven 4 el diagnóstico serológico fue un hallazgo durante la etapa de portador asintomático.

Tabla $\mathbf{N}^{\circ} 4$ Características generales de la muestra.

\begin{tabular}{|l|l|}
\hline Características generales de la muestra. & Detalles \\
\hline Ambulatorios & 58 \\
\hline Ingresados & 22 \\
\hline Edad promedio & 36 años \\
\hline $\begin{array}{l}\text { Tiempo medio desde el inicio de los síntomas hasta } \\
\text { el diagnostico. }\end{array}$ & 6 meses \\
\hline
\end{tabular}

Fuente: Elaboración propia

Figura 4. Características generales de la muestra 


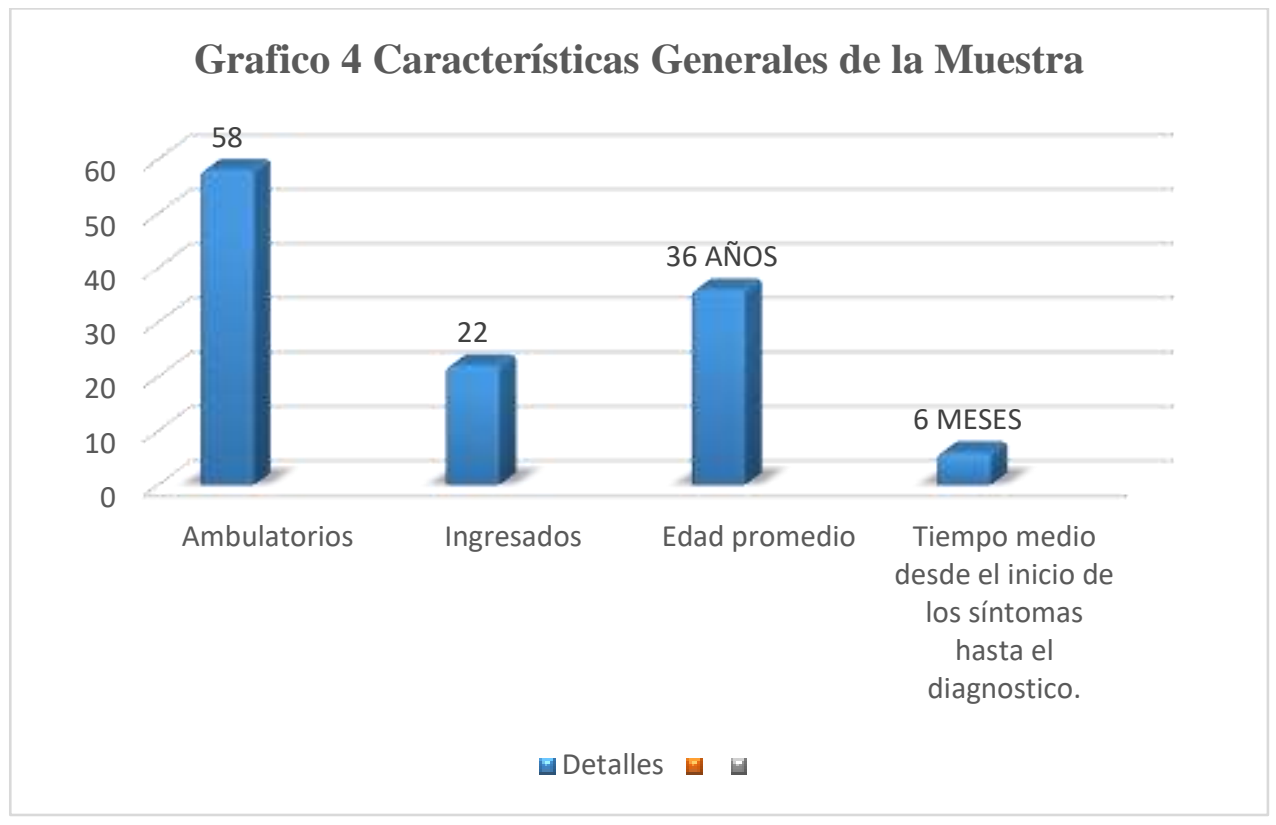

Fuente: Elaboración propia

\section{Análisis 4}

En la figura 4, se demuestra que en los 22 pacientes estudiados la edad promedio fue de 36 años y el tiempo medio de evolución desde el comienzo de los síntomas hasta la confirmación de la infección fue de 6 meses.

A consecuencia de la infección reciente por el VIH una pequeña parte de los pacientes pueden presentar síntomas tempranos seguidos de seroconversión; lo que se denomina retrovirosis aguda o infección aguda por el VIH, estos casos se incluyen en la categoría A de la clasificación de 1993 del Centro de Control de Enfermedades (CDC) de los Estados Unidos.

La retrovirosis aguda tiene varios cuadros clínicos, el más frecuente y mejor caracterizado es un síndrome que recuerda la Mononucleosis infecciosa. Otras formas son la Meningoencefalitis aséptica, parálisis facial periférica y diarreas entre las más frecuentes.

La Tabla 5 muestra los síntomas generales que motivaron el ingreso de los 22 casos que estudiamos. Un cuadro parecido a la Mononucleosis infecciosa fue diagnosticado en 9 pacientes (40.9\% del total de pacientes ingresados), con fiebre de inicio agudo, linfadenopatía, faringitis y hepatoesplenomegalia, sin síntomas previos, lo cual se interpretó como una infección primaria por el VIH al recibirse la confirmación serológica. El virus de la inmunodeficiencia humana aguda después de un periodo de incubación que tiene lugar pocos días o pocas semanas después de la exposición al virus de la inmunodeficiencia, la mayoría de los individuos infectados presentan una enfermedad aguda semejante a la gripe, la inyección aguda por virus de la inmunodeficiencia humana es un síndrome muy 
heterogéneo. Los individuos que presentan síntomas más severos durante la infección aguda tienden a progresar más rápido a SIDA, los síntomas clínicos de la infección aguda por VIH1 se descubrieron inicialmente en 1985 como una enfermedad parecida a la mononucleosis infecciosa.

Los síntomas más comunes son fiebres, erupción cutánea maculopapular, ulceras orales linfodenopatías, artralgias, faringitis, malestar, pérdida de peso, meningitis aséptica y mialgias. $* * * 9$ En un estudio publicado recientemente por Hecht y cols. (25) los síntomas de fiebre y erupción cutánea (especialmente en combinación) seguido de las ulceras orales y la faringitis tuvieron el mayor valor de pre- dicción para el diagnostico de la infección aguda por el virus de la inmunodeficiencia. En otro estudio efectuado por Daar y cols. Los mejores factores de pre- dicción de la infección aguda por virus de la inmunodeficiencia fueron la fiebre, la erupción cutánea, la mialgia y la sudoración nocturna. Y

En resumen, el paso más importante en el diagnóstico de la infección aguda por el VIH-1 es su inclusión en el diagnóstico diferencial, por tanto, la sospecha clínico de infección aguda por VIH-1 simplemente requiere la realización de una prueba de anticuerpo contra VIH-1 y probablemente la repetición de la determinación de la carga viral de VIH-1. Varias series han reportado la retrovirosis aguda entre 53-93\%, lo cual tiene una gran significación puesto que el manejo terapéutico precoz se relaciona con mejor pronóstico. *”**

Consideramos que los diagnósticos realizados han sido, en general, en etapas más avanzadas de la enfermedad lo cual pudiera estar en relación con dificulta- des en el pesquisaje a nivel primario de salud; con bajos índices de sospecha de esta infección o con formas clínicas complejas como son las neurológicas y las psiquiátricas.

De esta manera explicamos la mayor frecuencia de aquellos síntomas que aparecen en el curso de la enfermedad entre los que se destacan: la pérdida de peso y la linfadenopatía persistente por 3 meses o más en el $58.3 \%$ de esta serie: el decaimiento en el $50 \%$ y las diarreas crónicas en el $41.6 \%$. La linfadenopatía es un hallazgo frecuente y suele aparecer desde estadios muy tempranos de la enfermedad; puede ser generalizada, pero los ganglios cervicales, axilares y occipitales son los más encontrados. *”

En nuestra serie la localización cervical apareció en los 12 pacientes con adeno- patías (100\%), los axilares en 7 (33\%) y los inguinales en $1(8.3 \%)$. 
Tabla $\mathbf{N}^{\circ} 5$ Hallazgos Clínicos Generales.

\begin{tabular}{|l|l|l|}
\hline Sintomatología & Número de casos & $\%$ \\
\hline Linfadenopatia & 12 & 58.3 \\
\hline Perdida de peso & 12 & 58.3 \\
\hline Decaimiento & 11 & 50 \\
\hline Diarrea & 9 & 41.6 \\
\hline Fiebre & 9 & 41.6 \\
\hline Dolor abdominal & 7 & 33.3 \\
\hline Cefalea & 6 & 25 \\
\hline Depresion & 3 & 16 \\
\hline
\end{tabular}

Fuente: Elaboración propia

Figura 5. Hallazgos clínicos generales

Figura 5 Hallazgos Clínicos Generales

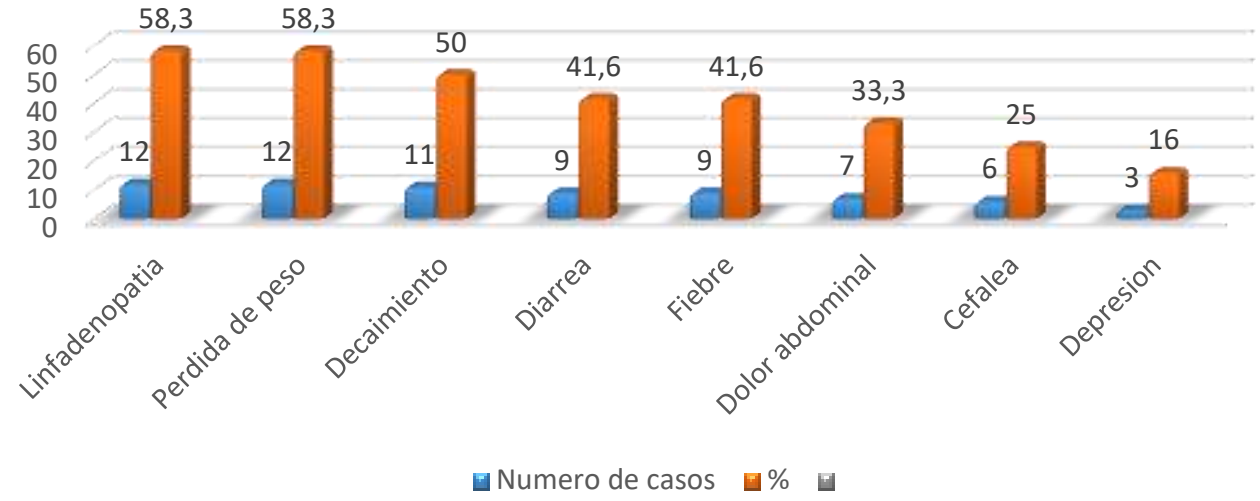

Fuente: Elaboración propia

\section{Análisis 5}

En este gráfico se explica que la mayor frecuencia de síntomas que aparecen en el curso de la enfermedad se destaca, la pérdida de peso. Linfadenopatía con porcentajes que corresponde al $58.3 \%$, decaimiento que corresponde al $50 \%$ y las diarreas crónicas en el 41.6\%, síntomas más relevantes referidos en el gráfico. 
Las causas de diarreas en esta enfermedad pueden ser múltiples; por lo común es crónica y se acompaña de morbilidad notable, consunción y caquexia.?" 2 Los gérmenes causales incluyen bacterias patógenas, virus, micobacterias y pro- tozoarios, con afectación entre el 44-85\% de estos pacientes. Nuestra casuística recoge diarreas en un $41.6 \%$ (9 casos) y en 2 de ellos se diagnosticó infección por Giardia Lamblia. ¿Varios estudios han reportado que la infección por este protozoario no difiere entre los pacientes VIH positivos y la población general 2? 20.31) Presentaron baja frecuencia de aparición la cefalea y la tos, ambas en solo 6 casos. En grandes estudios clínicos, los síntomas respiratorios han sido raros en los estadios iniciales y se asocian con la progresión de la enfermedad y la disminución de los linfocitos $\mathrm{CD} 4+.2$

El síndrome ansioso depresivo fue la causa de ingreso en 3 pacientes (16\%). Treisman y Schwatz plantean que más del $20 \%$ de los pacientes infectados por el VIH pueden sufrir depresión debido a un daño neurológico causado por el propio virus o posterior al conocimiento del diagnóstico. Los estudios complementarios que se analizaron (Tabla 6) fueron Hematocrito, Conteo de leucocitos, Eritrosedimentación, Transaminasa glutámico oxalacética (TGO), Transaminasa glutámica pirúvica (TGP) y Deshidrogenasa láctica (LDH).

Los valores promedios de estas variables estuvieron dentro de parámetros normales. Las anormalidades hematológicas más frecuentes en la infección por el VIH son las citopenias, observadas en estadios avanzados de la enfermedad, en nuestra serie consideramos a 3 pacientes con diagnósticos tardíos, pero no se encontró anemia ni leucopenia.

El ultrasonido abdominal fue útil en la confirmación de hepatomegalia y esplenomegalia. La radiografía de Tórax demostró proceso de condensación inflamatorio en un paciente con tos, fiebre y disnea.

La biopsia por aspiración con aguja fina (BAAF) de ganglio fue realizada en 11 casos, 10 con hiperplasia linfoide y 1 con diagnóstico de Linfoma no Hodakin.

La definición de Síndrome de Inmunodeficiencia Humana (SIDA) por el CDC de

1993 incluye todos los individuos infectados por el VIH que tienen menos de 200 células CD4+ por milímetro cúbico de sangre además de 26 condiciones clínicas que afectan a las personas con enfermedad por el VIH avanzada. La mayoría de estas condiciones son infecciones oportunistas, encontrando también neoplasias y afecciones neurológicas.

Tabla $\mathbf{N}^{\circ}$ 6: Hallazgos de Laboratorios. 


\begin{tabular}{|l|l|}
\hline Complementarios & Resultados promedios \\
\hline Hematocrito & 0.37 \\
\hline Leucocitos & 6.8 \\
\hline VSG & 57 \\
\hline TGO & 39.4 \\
\hline TGP & 37 \\
\hline LDH & 343 \\
\hline Ultrasonido abdominal & normal \\
\hline Baaf de ganglio & Hiperplasia linfoidea \\
\hline
\end{tabular}

Fuente: Elaboración propia

Figura 6. Hallazgos de laboratorio

Figura 6. Hallazgos de Laboratorio

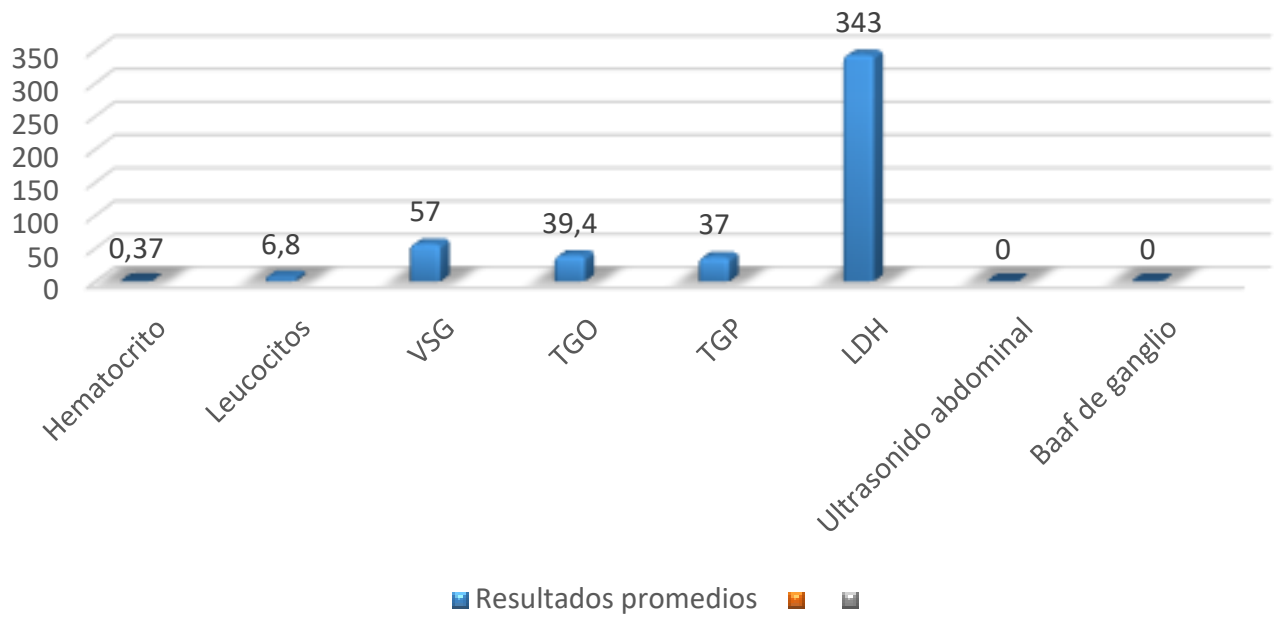

Fuente: Elaboración propia

\section{Análisis}

Como se aprecia en el gráfico 6, los valores promedios de estas variables estuvieron dentro de parámetros normales. 
A ninguno de los pacientes estudiados se les realizó conteo de CD4+ en el momento del diagnóstico, debido a que es un estudio que se realiza en el IPK como parte de la evaluación posterior del paciente, por lo que no contamos con un parámetro decisivo para clasificarlos. Encontramos 9 enfermos con patologías que definen SIDA (Categoría C de la clasificación de 1993): Tuberculosis pulmonar (2 casos), el síndrome de adelgazamiento por el VIH (2 casos), Linfoma no Hodgkin tipo Burkitt (1), Candidiasis esofágica, traqueal y pulmonar (1), Neumonía por Pneumocystis carinii ( 2 casos) y Neurotoxoplasmosis (1 caso).

La tuberculosis pulmonar (TB) fue un diagnóstico previo al ingreso en un paciente que se admitió en unidad de Quemados, bajo tratamiento antituberculoso en segunda fase, donde las condiciones clínicas y epidemiológicas hacían posible la hipótesis de una infección por VIH previo a la TB.

Se hizo diagnóstico de Tuberculosis pulmonar en un paciente con lesiones radio- lógicas en bases pulmonares, de 2 meses de evolución, sin mejoría con antibacterianos usuales y con baciloscopia positiva.

La "reemergencia" de la tuberculosis desde mediados de la década de los 80, ha estado de forma exponencial ligada a la epidemia de la infección por el virus de la inmunodeficiencia humana tipo |. Particularmente en naciones asiáticas y del África Subsahariana se evidencia un aumento de la incidencia a más de 300 casos por 100000 habitantes, agrupándose en estas áreas cerca de $50 \%$ de las personas coinfectadas. En la última década del pasado siglo 8000 000 de casos de tuberculosis fueron atribuibles al SIDA, con alrededor de 2900000 muertes. 96, 37 La superposición de poblaciones infectadas por VIH y de poblaciones infectadas por Mycobacterium tuberculosis favorece la interacción entre ambos patógenos, de tal manera que la coinfección-coenfermedad, ha desencadenado modificaciones no despreciables en el comportamiento clínico y epidemiológico de estos procesos patogénicos.

Cuba, con patrones de incremento de la infección por el VIH y de desarrollo del SIDA diferente al resto de los países del tercer mundo, no está exenta de esta problemática. El primer caso de tuberculosis asociada al VIH se reportó en mayo de 1992, tratándose de una tuberculosis pleural que curó con la terapéutica impuesta. Con el surgimiento de nuevos casos, se ha observado gran variabilidad en la expresión clínica de la tuberculosis y en el estado inmunológico de los pacientes que desarrollan esta dolencia, hechos que en cierta medida se apartan de lo documentado en la literatura.

Girardi y otros del Grupo Italiano de Estudio de la Tuberculosis y el SIDA, en 67 pacientes sin terapia antirretroviral, reportaron a la tuberculosis como primera enfermedad indicadora en $58.0 \%$. Las características clínicas más sobresalientes en los enfermos coincidieron con las descritas por la literatura clásica. Martin G des- taca la alta frecuencia de los síntomas tradicionales de la tuberculosis en los enfermos SIDA. Sin embargo, la hemoptisis no resultó 
usual en nuestra serie donde la sintomatología fue típica de proceso inflamatorio del pulmón. $1-?$

El síndrome de adelgazamiento por el VIH ("wasting syndrome") caracterizado por pérdida de peso mayor del 10\% del peso corporal previo, diarrea crónica por más de 30 días, debilidad crónica y fiebre intermitente o constante por más de 30 días, fue constatado en 2 pacientes donde se habían planteado los diagnósticos de síndrome de mala absorción intestinal y parasitismo rebelde a tratamiento

Se plantea que los mecanismos potenciales relacionados con este síndrome son: pérdida de energía a nivel celular por elevado metabolismo energético, disminución de la ingestión de fuentes de proteínas, uso ineficiente de sustratos energéticos y factores hormonales. Hay diferencias bien establecidas del síndrome en hombres y mujeres en cuanto a la distribución de la pérdida de peso, en los hombres hay pérdida pareja de la grasa y de la masa muscular, mientras que en las mujeres la pérdida muscular es en estadios más avanzados de la enfermedad. En los 2 pacientes de nuestra serie con síndrome de desgaste por VIH, un hombre y una mujer, no observamos diferencias sustanciales en la forma de adelgazamiento que permitan hacer comparaciones.

El Linfoma no Hodgkin de alto grado, de células pequeñas no hendidas tipo Burkitt fue el diagnóstico anatomopatológico de un paciente fallecido como consecuencia del SIDA, cuya confirmación serológica fue postmortem. Este enfermo comenzó 3 meses antes con dolor abdominal y se constata una masa tumoral en hipogastrio.

Luego apareció ptosis palpebral, diplopia, cefalea, dolor facial y dificultad para la marcha; 1 biopsia del tumor abdominal, tomada por laparotomía planteó un Linfoma Hodgkin variedad celularidad mixta y una BAAF de ganglio maxilar informó un Linfoma no Hodgkin de alto grado de malignidad.

De los linfomas no Hodgkin diagnosticados en los pacientes VIH positivos el 30- 40\% corresponde a Linfoma de Burkitt por lo que se considera el linfoma más frecuente en los pacientes con SIDA. *\% A este paciente se le hizo un diagnóstico tardío lo que permitió una rápida progresión de la enfermedad con poco tiempo de supervivencia.

El Centro para el Control de Enfermedades (CDC) de Atlanta hace una revisión de la definición del SIDA en el período de 1985 a 1987 para incluir a pacientes con el diagnóstico de linfoma no Hodgkin (LNH) de células B agresivos. La incidencia de LNH ascendió en un desarrollo casi paralelo con la epidemia del SIDA y se diagnosticó en 2 a $3 \%$ de los casos recién identificados. 
El número de personas con SIDA diagnosticadas con LNH fue en rápido aumento hasta 1995, año en que resulta disponible el tratamiento antirretroviral de gran actividad (TARGA). En general, el entorno clínico y la respuesta al tratamiento de los pacientes con linfoma relacionado con el SIDA son muy diferentes de los pacientes sin VIH con linfoma. En aproximadamente $90.0 \%$ de los casos se demuestra la presencia de productos de la infección por virus Epstein Barr (VEB),32 como son la proteína de membrana del virus -LMP1. El linfoma presentó una localización extraganglionar en $70 \%$ de los pacientes, lo que coincide con los registros revisados. Kaplan y otros al igual que Levine y otros encontraron que la participación extranodal está relacionada con una disminución de la supervivencia en pacientes con diagnóstico de linfoma y SIDA estudiados por ellos. 50.51

Histológicamente existió predominio de linfoma no Hodgkin, subtipo células B grandes difusas; iguales resultados se recopilan en otros ensayos. Se describen un comportamiento agresivo de este tipo de tumor y pobre respuesta al tratamiento, si se compara con los pacientes no VIH. El subtipo histológico constituye el factor individual más importante en los LNH relacionados con SIDA. El patrón arquitectónico difuso formado mayoritariamente por células grandes, tiene un pro- nóstico desfavorable, por lo que, en opinión de los autores del presente trabajo, el predominio de este subtipo histológico guardó estrecha asociación con la evolución y mortalidad de los pacientes. Se pudo demostrar una relación estadísticamente significativa $(\mathrm{p}<0.05)$ entre el grado de inmunodepresión del paciente y el estadio de evolución del tumor en el momento del diagnóstico, esta relación la describen también otros autores como Kaplan y otros quienes reportan además que estos factores tienen valor pronóstico en la supervivencia del paciente y en la respuesta al tratamiento oncológico. !: 2. $\% .5$

La candidiasis diseminada se diagnosticó en un paciente con historia de esofagitis moniliásica tratado en el servicio de Gastroenterología por 2 meses, que inicia con manifestaciones respiratorias, fiebre elevada y lesiones radiológicas diseminadas que en los hemocultivos y cultivos de secreciones bronquiales y pulmonares obtenidas por broncoscopio confirmó el diagnóstico de candidiasis junto a la confirmación serológica de infección por VIH. La infección por Candida albicans se suele manifestar en la boca, garganta, prepucio del pene, vagina y los pliegues húmedos en los dobleces de la piel Es una señal temprana del debilitamiento del sistema Inmune y se ve primero cuando el nivel de células T oscila entre 200 y 500 y puede ocurrir incluso en casos de niveles altos de células T. La infección de Candida del esófago ocurre cuando la enfermedad del VIH se encuentra más avanzada. En nuestro caso hubo respuesta favorable al tratamiento antimicótico por 3 semanas, a pesar de persistir el dolor de garganta

Dos pacientes con manifestaciones respiratorias de más de 1 mes de evolución, dadas por tos, disnea a mínimos esfuerzos y expectoración escasa con estudios serológicos confirmados 
de infección por VIH, han sido incluidos con la sospecha de neumonía por Pneumocystis carinii. En la evolución de uno de los casos hubo un distress respiratorio que a pesar de tratamiento en unidad de cuidados intensivos (UCI).

La enfermedad invasiva por neumococo se incrementa en asociación con el VIH. Un gran aumento de bacteriemia por neumococo y una mortalidad elevada por neumonía neumocócica se han encontrado en pacientes jóvenes seropositivos. 6 Las personas infectadas por el VIH padecen de neumonía neumocócica con una frecuencia de 5 a 15 veces mayor que las personas VIH seronegativas. Strepto- coccus pneumoniae es la bacteria que más frecuentemente causa neumonía en Pacientes VIH seropositivos.

Un estudio de 63 pacientes VIH/SIDA con infecciones respiratorias agudas (IRA) ingresados en el Hospital del Instituto de Medicina Tropical "Pedro Kourí" en el Período comprendido de enero a agosto de 1997 procesó un total de 545 muestras: 153 esputos, 158 hemocultivos, 110 exudados faríngeos, 61 exudados nasa- les y 63 exudados óticos. Los agentes bacterianos más frecuentes causantes de IRA del total de cepas aisladas fueron: Pseudomonas aeruginosa (29.65\%), Staphylococcus aureus (27.14 \%) y Streptococcus pneumoniae (9.04\%). Los microorganismos más frecuentemente asociados con bacteriemias fueron: Staphy- lococcus epidermidis (38.70\%), Staphylococcus aureus (16.13\%), Pseudomonas Aeruginosa (12.90 $\%)$, y Salmonella tipo b (12.90\%). *"

Se ha comentado la ocurrencia de infecciones dobles en el tracto respiratorio, donde uno de los patógenos propuestos es el M. pneumoniae asociado funda- mentalmente a virus; se observa, además, que las sepsis por gramnegativos asociada a la injuria pulmonar producida por los M. pneumoniae o M. fermentans es uno de los factores predisponentes para el desarrollo del síndrome de distrés respiratorio del adulto o enfermedad sistémica con fallo multiorgánico. Otras especies de micoplasmas como M penetrans, M. pirum, M. genitalium y M. hominis han sido repetidamente aislados en pacientes con SIDA. En nuestros casos, no contamos con evidencia de infección bacteriana y la respuesta al tratamiento con sulfaprim endovenosos fue favorable hasta su traslado al IPK. \%-

La neurotoxoplasmosis fue el diagnóstico de un paciente que ingresa por pérdida marcada de peso, fiebre, cefalea, diarreas, anemia y alteraciones de la memoria y el cálculo. En la resonancia magnética nuclear se evidenció lesión en región parietal izquierda hipointensa de 15x19mm rodeada de edema. La Toxoplasmosis cerebral es la causa más frecuente de lesión cerebral en pacientes SIDA, pero Ofrece en ocasiones, dudas diagnósticas con el Linfoma y la Leucoencefalopatía Multifocal progresiva, lo que requeriría de estudios histológicos.

El análisis realizado en esta serie ha permitido conocer elementos clínicos asociados con mayor frecuencia a la infección por el VIH, formas de presentación no frecuentes de la enfermedad y elementos de orden epidemiológico que permiten caracterizar determinados 
grupos de riesgo, todo lo cual es de gran importancia para el médico que se enfrenta a la atención del paciente en un hospital.

\section{Conclusiones.}

- En el periodo comprendido entre los años 2002 y 2007 se diagnosticaron 80 infecciones por el virus de la inmunodeficiencia humana, la mayoría de forma ambulatoria.

- Todos los casos fueron confirmados por Western Blot en el Laboratorio de Referencia Nacional de la Defensa Civil (LISIDA).

- Los elementos epidemiológicos de mayor importancia fueron: el sexo masculino y la procedencia de la Ciudad de la Habana.

- Los síntomas clínicos más frecuentes fueron: linfadenopatías (58.3\%), pérdida de peso $(58.3 \%)$, astenia $(50.0 \%)$ y diarreas $(41.3 \%)$.

- Se diagnosticaron 9 pacientes con retrovirosis aguda, 9 en estadio SIDA y en periodo de portador asintomático.

- Se recomienda fomentar estrategias educativas a través de todos los medios disponibles, especialmente las intervenciones directas con la población, utilizando las potencialidades del sistema nacional de salud, familiar para la evitar el contagio y para hacer diagnóstico en fases iniciales de la infección

- Tener un alto índice de sospecha de infección por el virus de la inmunodefi- ciencia humana en pacientes con síndrome adénico, pérdida de peso, astenia y diarreas de causa no demostrada.

- Conocer y cumplir las medidas universales de bioseguridad para prevenir el contagio del virus en el personal hospitalario, conociendo que toda persona que requiera de nuestro servicio tiene derecho a ser atendida con calidad técnica y elevada sensibilidad humana.

\section{Referencias bibliográficas.}

De Quesada Ramírez. Galbán García, Cádiz Lahens. SIDA. Infección- enfermedad por el virus de la inmunodeficiencia humana. Editorial Científico Técnica, 1987.

Pérez Ávila Jorge, Villa verde Añe Biarda. Pautas para la atención clínica de las personas infectadas con el VIH o enfermos de SIDA. Programa Nacional de Prevención y Control del SIDA, Ministerio de Salud Pública, 1995

Plan Estratégico Nacional ITS/VIH/SIDA 2001-2006. MINSAP 
The Global HIV and AIDS Epidemic, 2001. Morbility and Mortality Weekly Report. Vol. 50 , N. 21, junio 2001

La Epidemia SIDA sigue teniendo proporciones catastróficas. Revista Panamericana de Salud Pública. Vol. 7, N. 2. 127-128, feb. 2000.

Sida global: verdades y mentiras: herramientas para luchar contra la pandemia del siglo XXI. Irwin, Alexander; Millen, Joyce; Fallows, Dorothy; Aguiar González, Fernando. Barcelona. Ediciones Paidós Ibérica, S.A. ISBN 84-493-1626-X

M. W. Makgoba (2000). «HIV/AIDS: the peril of pseudoscience». Science 288 (5469): 1171.

M. W. Makgoba (2002). «Politics, the media and science in HIV/AIDS: the peril of pseudoscience». Vaccine 20 (15): 1899-1904.

Fichil, M.A.: "An introduccion to the clinical spectrum of AIDS" en Textbook of AIDS Medicine. Editores: Broder, S., Merigan, T.C.; Bolognesi, D: Wil- liams $€$ Wilkins. Baltimore. 1994. 149.

El sida y sus metáforas. Susan Sontag. Barcelona. Edita: El Aleph, 1989. 104 p. ISBN 8476690851

Kahn JO, Walker BD. Acute Human Immunodeficiency Virus Type 1 Infec- tion. N Engl J Med 1998; 339: 33-39

Carr A; Cunningham P; Kelleher A; Cooper D. Diagnosis and treatment of primary HIV infection. The Journal of HIV Combination Therapy, Vol 2, N. 3, Agosto 1997: 49-56

Fischl M: Clinician's Manual on tha Mnagement of HIV. Second Edition. 1998. Glaxo Welcome- HIV

Machado, J.A. et al.: Isolation and purification of the transmembrane gp41 VIH-1 Recombinant protein synthetized in E. coli. 1er Congreso Iberoamericano de Biotecnología. Libro de Resúmenes. (S05-050). La Habana, Cuba, 1989 
Albertini, a. Et al.: Evaluation of the ANTI- HIV Ultramicoelisa (UMELISA) kit with the Ultramicroanalyte Sistem (SUMA). Clinical Chemistry, 36: 1091. 1990

Díaz Torres, H. M, Rodríguez García, O., Sánchez Álvarez, M. Retrovirosis aguda. Informe de un caso. Rev. Cubana MED 34(1):63-66, enero- abril,

Vanhems P, Alland R, Cooper DA et al: Acute HIV-1 disease As mononu- cleosis- like illness: is the diagnosis too restrictive? Clin Infect Dis, 1997, 24, 965.

Hecht FM, Bush MP, Rawal B, Webb M, Rosenberg E, Swanson M et al: Use of laboratory test and clinical symptoms for identification of primary HIV infection. AIDS 2002,16:11191129. Http/amadeo

Daar E, Little S, Pitt J, Santangelo J, Ho P, Harawa N, et al: Diagnosis of primary HIV-1 infection. Los Angeles County primary HIV infection Recru- itment Network. Ann Intern Med 2001,134:25-29

Framm SR, Soave R: Agentes productores de diarrea. Clínicas Médicas de Norteamérica, Vol 2, 1997. Traducción de la edición en inglés Vol 81, N. 2: 417-438

Bartlett JG, Bitsos PC, and Sears CL: AIDS enteropathy. Clin Infect Dis 15:726, 1992

Liesenfel O, Schneider T, Schmidt W et al: Culture of intestinal biopsy specimens and stool culture for detection of bacterial enteropathogens in Patiens infected with HIV. J Clin Microbiol 33: 745, 1995

Laughon BE, Druckman DA, and Vernon a: Prevalence of enteric patho- gens in homosexual men with and without acquired immunodeficiency syn- drome. Gastroenterology 94: 948, 1998

Smith PD, Acute infections diarrhea: Infections diarrheas in patients with AIDS. Gastroenterol Clin North Am 22: 535, 1993

Centers for Disease Control: Recomendations for prophilaxis againts Pneumocystis carinii pneumonia for adults and adolescents infected with HIV.MMWR 41:1-11, 1992 
Treisman G, Schwartz J: Psychiatric disorders. En: 2000-2001 Medical Managament of HIV infection. John Hopkins University, Department Of. In- fectious Diseases, 2000

Coyle TE: Complicaciones hematológicas de la infección por VIH y del SI- DA. Clínicas Médicas de Norteamérica, Vol 2: 439-461,1997

Gallant JE: Strategies for long- term success in the treatment of HIV infec- tions. JAMA, Vol.28, March 8, 2000, pp 1329-1334

Bates JH, Stead WWW. The history of tuberculosis as a global epidemic. Med Clin Noth Am 1993; 77(6):1205-17.

Bentwich Z, Maartens G, Torten D, Lal AA, Lal RB. Concurrent infections and HIV pathogenesis. AIDS 2000; 14:2071-81.

Del Amo J, Malin AS, Pozniak A, de Cock KM. Does tuberculosis acceler- ate the progression of HIV disease? Evidence from basic science and epi- demiology. AIDS 1999; 13(10):1159-64.

Nolan CM. Nosocomial multidrug-resistant tuberculosis. Global spread of The third epidemic. J Infect Dis 1997; 176(3):748-51.

Torres R, Fiol J, Carreras L, Pérez J, Hernández O, Marrero A, et al. La in- fección por el virus de la inmunodeficiencia humana y la tuberculosis en Cuba. Bol Ofic Sanit Panam 1995; 119(1):66-73

Girardi E, Palmieri F, Cingolani A, Ammassari A, Petrosillo N, Gillini L, et al. Changing clinical presentation and survival in HIV-associated tuberculosis after highly active antiretroviral therapy. J Acquir Immune Defic Syndr 2001; 26(4):326-31.

Martin G, Lazarus A. Epidemiology and diagnosis of tuberculosis: recogni- tion of at-risk patients is key to prompt detection. Postgrad Med 2000; Ga- tell Artigas JM, Miró Meda JM, Pumarola Suñe. Infecciones causadas por retrovirus humanos: SIDA, En Farreras- Rozman: Tratado de Medicina In- terna. 14 ediciones. 2000 
Blanchard C, Gazzard BG: Natural history and prognosis of diarrhea of un- known cause in patient with AIDS Am ¡Med 92:404, 1992

Mulligan K, Grunfeld C, Hellerstein MK, Neese RA, Schambelan M. Ana- bolic effects of recombinant human growth hormone in patients with wast- ing associated with human immunodeficiency virus infection. J Clin Endo- crinol Metab 1993; 77:956-962. [Abstract

Dobs AS, Dempsey MA, Ladenson PW, Polk BF. Endocrine disorders in men infected with human immunodeficiency virus. Am J Med 1988; 84:611- 616. [CrossRef][Medline]

Gerard L, Galicier L, Boulanger E. Improved survival in HIV-related Hodgkin's lymphoma since the introduction of highly active antiretroviral therapy. AIDS 2003; 17(1):81-7

International Collaboration on HIV and Cancer. Highly active antiretroviral therapy and incidence of cancer in human immunodeficiency virus-infected adult. J Natl Cancer Inst 2000; 92(22):1823-30.

Hoffmann C, Wolf E, Fátkenheuer G. Response to highly active antiretrovi- ral therapy strongly predicts outcome in patients with AIDS-related lym- phoma. AIDS 2003; 17(10):1521-9

Kirk O, Pedersen C, Cozzi-Lepri A. Non-Hodgkin lymphoma in HIV- infected patients in the era of highly active antiretroviral therapy. Blood 2001; 98:3406-12

Kaplan LD, Abrams DI, Feigal E. AIDS's associated non Hodgkin lym- phoma in San Francisco. JAMA 1999; 261:5:719-24.

Besson C, Goubar A, Gabarre J. Changes in AIDS-related lymphoma since the era of highly active antiretroviral therapy. Blood 2001; 98:2339

Herida M, Mary-Krause M. Incidence of non-AIDS-defining cancers be- fore and during the highly active antiretroviral therapy era in a cohort of human immunodeficiency virusinfected patients. J Clin Oncol 2003; 21:3447-53. 


\section{PARA CITAR EL ARTÍCULO INDEXADO.}

Velázquez Lores, L., Andrade Tacuri, G., Triana Santana, G., \& Ayala Briones, J. (2019). Caracterización de la infección por el virus de la inmunodeficiencia humana en el Hospital Hermanos Ameinjeiras, $2002 \quad$ - 2007. Ciencia Digital, 3(2.1), 156-180. https://doi.org/10.33262/cienciadigital.v3i2.1.437

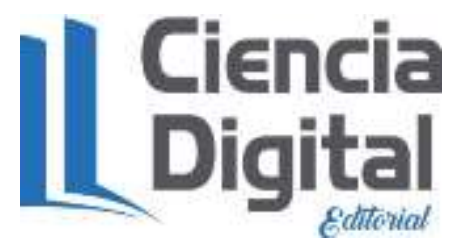

El artículo que se publica es de exclusiva responsabilidad de los autores y no necesariamente reflejan el pensamiento de la Revista Ciencia Digital.

El artículo queda en propiedad de la revista y, por tanto, su publicación parcial y/o total en otro medio tiene que ser autorizado por el director de la Revista Ciencia Digital.
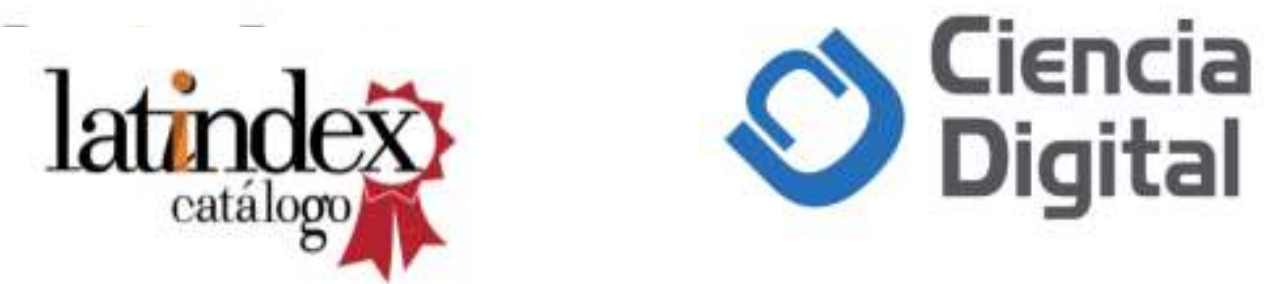